\title{
ASYMPTOTIC ABELIANNESS OF INFINITE FACTORS
}

\author{
BY
}

\author{
M. S. GLASER( $\left.{ }^{1}\right)$
}

ABSTRACT. Studying Pukánszky's type III factor, $M_{2}$, we show that it does not have the property of asymptotic abelianness and discuss how this property is related to property $\mathrm{L}$. We also prove that there are no asymptotic abelian $\mathrm{II}_{\infty}$ factors. The extension (by ampliation) of central sequences in a finite factor, $N$, to $M \otimes N$ is shown to be central. Also, we give two examples of the reduction (by equivalence) of a central sequence in $M \otimes N$ to a sequence in $N$. Finally, applying the definition of asymptotic abelianness of $C^{*}$-algebras to $W^{*}$ algebras leads to the conclusion that all factors satisfying this property are abelian.

1. Introduction. A new algebraic invariant has recently been introduced into the study of $W^{*}$-algebras. This is the property of asymptotic abelianness of factors. It was first used by physicists in their study of $C^{*}$-algebras and defined by means of the uniform topology. Sakai [11] extended the concept to $W^{*}$-algebras by using the strong topology. This extension immediately proved to be useful in the classification of $W^{*}$-algebras. Sakai showed that the infinite tensor product of a II factor is asymptotically abelian and that there are nonasymptotically abelian $\mathrm{II}_{1}$ factors. Thus, given a nonasymptotically abelian $\mathrm{II}_{1}$ factor, it is easy to construct a different $\mathrm{II}_{1}$ factor. The other finite type II factors and all finite type I factors were then classified according to their asymptotic abelianness ([4], [11], and [17]). However, it proved to be more difficult to show infinite factors were either asymptotically abelian or not asymptotically abelian. Willig [15] proved that the $I_{\infty}$ factor was not asymptotically abelian, but the other cases, up to now, were not known.

In this paper, we study asymptotic abelianness of type $\mathrm{II}_{\infty}$ and type III factors. We prove that the type III factor of Pukánszky [10] is not asymptotically abelian and that none of the $\mathrm{II}_{\infty}$ factors are asymptotically abelian. These results suggest that there may not be any asymptotically abelian infinite factors. The truth of this suggestion is not yet known. However, belief in it would be corroborated if it could be shown that Powers' factors [9] are not asymptotically abelian. We

Received by the editors September 22, 1970 and, in revised form, July 12, 1971.

AMS (MOS) subject classifications (1970). Primary $46 \mathrm{Ll} 10$.

Key words and phrases. Asymptotic abelianness, type III factor, type II factor, property L, central sequence, equivalent sequences, trivial sequence, *-automorphism, invariant state, strong convergence, uniform convergence.

(1) This paper is part of the author's doctoral dissertation at the University of Pennsylvania. The work was supervised by Professor Shôichirô Sakai. 
will discuss why these factors are crucial examples by showing the relationship between asymptotic abelianness and property $\mathrm{L}$.

We also investigate two other concepts which are related to the property of asymptotic abelianness -- central sequences and uniform asymptotic abelianness. As for the latter, we show that there are no noncommutative uniformly asymptotically abelian $W^{*}$-algebras. The former is studied in two ways -- we give sufficient conditions for the reduction of a central sequence in a $W^{*}$-tensor product and sufficient conditions for its extension.

The following properties are important to all sections of this paper. (Properties which are used briefly will be defined when they are needed.)

Definition 1.1. Let $M$ be a factor. $M$ is called asymptotically abelian if there is a sequence, $\left(\rho_{n}\right)$, of $*$-automorphisms on $M$ such that strong $\operatorname{limit}\left[\rho_{n}(A), B\right]$ $=0$ for each $A, B \in M$, where $[X, Y]=X Y-Y X$ for $X, Y \in M$.

Definition 1.2. Let $M$ be a factor. A uniformly bounded sequence, $\left(A_{n}\right)$, of elements of $M$ is called a central sequence if strong limit $\left[A_{n}, B\right]=0$ for all $B$ $\in M$. A sequence $\left(A_{n}\right) \subset M$ is called *-central if $\left(A_{n}\right)$ is central and $\left(A_{n}^{*}\right)$ is central.

Definition 1.3. Let $M$ be a $W^{*}$-algebra. Two uniformly bounded sequences, $\left(A_{n}\right)$ and $\left(B_{n}\right)$, in $M$ are called equivalent if strong limit $\left(A_{n}-B_{n}\right)=0$. A sequence $\left(A_{n}\right)$ is called trivial if $\left(A_{n}\right)$ is equivalent to a sequence $\left(\lambda_{n} l\right)$ where $\lambda_{n} \in \mathbf{C}$ for each $n$.

The distinction between *-central and central sequences is only significant in the infinite case because if $M$ is a finite factor, every central sequence is *-central.

An important fact, which will be used later, is that any sequence equivalent to a central sequence is also central. Let $\left(A_{n}\right)$ be a central sequence in $M$ and $\left(B_{n}\right)$ a uniformly bounded sequence such that $\left(B_{n}\right)$ is equivalent to $\left(A_{n}\right)$. Then, for $X \in M$ and $x \in H$, the underlying Hilbert space,

$$
\begin{aligned}
\left\|\left[B_{n}, X\right] x\right\| & \leq\left\|\left[B_{n}-A_{n}, X\right] x\right\|+\left\|\left[\Lambda_{n}, X\right]_{x}\right\| \\
& \leq\left\|\left(B_{n}-A_{n}\right) X x\right\|+\|X\|\left\|\left(B_{n}-\Lambda_{n}\right) x\right\|+\left\|\left[\Lambda_{n}, X\right] x\right\|<3 \epsilon .
\end{aligned}
$$

2. The type III factor $M_{2}$. We begin this section by constructing Pukánszky's factor, $M_{2}$. Theorem 2.5 will prove that $M_{2}$ is not asymptotically abelian and thus give the first example of a continuous infinite factor which is not asymptotically abelian.

First, form the measure space $\left\{X_{0}, S_{0}, \mu_{0}\right\}$ where $X_{0}=\{0,1\}, S_{0}=$ the class of subsets of $X_{0}$, and $\mu_{0}(\{0\})=p, \mu_{0}(\{1\})=1-p(0<p<1 / 2)$. Let $\{X, S, \mu\}$ be the completion of the Cartesian product $\left\{X_{g \in \Phi_{2}} X_{g}, X_{g \in \Phi_{2}} S_{g}, X_{g \in \Phi_{2}} \mu_{g}\right\}$ where 
$\Phi_{2}$ is the free group on two generators, $g_{1}$ and $g_{2}$, and $X_{g}=X_{0}, S_{g}=S_{0}, \mu_{g}=\mu_{0}$ for all $g \in \Phi_{2}$. A point $x \in X$ is denoted $\left(x_{g}\right)$ where $x_{g}=0$ or 1 for all $g \in \Phi_{2}$.

Let $\Delta=\left\{x \in X \mid x_{g}=0\right.$ except for a finite number of $\left.g\right\} . \Delta$ is an abelian group under the operation $(x+y)_{g}=x_{g}+y_{g}(\bmod 2)$. Form a new group $\biguplus=$ $\left(\Delta \mid \Phi_{2}\right)$, the semidirect product of $\Delta$ and $\Phi_{2}$, with $\Phi_{2}$ inducing on $\Delta$ the automorphism, $T_{g_{0}}$, defined at the gth coordinate by $\left(T_{g_{0}} \alpha\right)_{g}=\alpha_{g_{0} g}$ for $g_{0} \in \Phi_{2}$, $a \in \Delta$. Thus, for $(\alpha, g),\left(\beta, g_{0}\right) \in\left(\Delta \mid \Phi_{2}\right)=乌_{\text {, }}$

$$
(\alpha, g)\left(\beta, g_{0}\right)=\left(T_{g_{0}} \alpha+\beta, g g_{0}\right), \quad(\alpha, g)^{-1}=\left(T_{g^{-1}} \alpha, g^{-1}\right)
$$

and $(0, e)$ is the identity where 0 is the identity in $\Delta$ and $e$ is the identity in $\Phi_{2}$. An element in $\varrho$ of the form $(\alpha, e)$ will be denoted $\alpha$ and $(0, g)$ will be denoted $g$.

$\mathcal{G}$ acts as a group of one-to-one mappings of $X$ onto itself by the rule $x \rightarrow$ $x a$ where, letting $x=\left(x_{g}\right)$ and $a=\left(a, g_{0}\right),(x a)_{g}=x_{g_{0} g}+a_{g}(\bmod 2)$. Since $\biguplus$ is also a group of automorphisms of $S$, for each $a \in \mathcal{G}$, we may define the translated measure $\mu_{a}(E)=\mu(E a)$, for $E \in S . \mu_{a}$ is absolutely continuous with respect to $\mu$, so the Radon-Nikodym derivative $d \mu_{a}(x) / d \mu$ exists.

Now, for $a \in \mathcal{G}$, let $H_{a}=L_{\mu}^{2}(X)$, the Hilbert space of all $\mu$-square integrable functions on $X$ and form $H=\Sigma_{a \in \varrho} \bigoplus H_{a}$. An element $F \in H$ is a complexvalued function, $F(a, x)\left(a \in \varrho_{,}, x \in X\right)$ satisfying the condition $\Sigma_{a \in \varrho} \int_{X}|f(a, x)|^{2} d \mu$ $<\infty$. The inner product on $H$ is defined by

$$
(F, G)=\sum_{a \in \mathfrak{Q}} \int_{X} F(a, x) \overline{G(a, x)} d \mu
$$

for $F, G \in H . M_{2}$ is then defined as the algebra over $H$ generated by the operators

$$
\left(\bar{U}_{a_{0}} F\right)(a, x)=\left(d \mu_{a_{0}}(x) / d \mu\right)^{1 / 2} F\left(a a_{0}, x a_{0}\right), \quad\left(\vec{L}_{\phi(x)} F\right)(a, x)=\phi(x) F(a, x)
$$

where $a, a_{0} \in \mathcal{G}, F \in H$, and $\phi(x)$ is a complex-valued measurable function. Every operator in $M_{2}$ is of the form $A=\Sigma_{a \in \mathcal{G}} \bar{L}_{\phi_{a}(x)} \bar{U}_{a}$ where $\phi_{a}(x)$ is bounded and measurable for $a \in \mathfrak{G}$. The infinite sum is defined by the strong operator topology. We will denote $(A)_{a} \equiv \phi_{a}(x)$.

We will use the following lemmas in this section and in $\$ 5$. For proofs, the reader is referred to [10].

Lemma 2.1. If $(A)_{a} \equiv \phi_{a}(x)$ and $(B)_{a} \equiv \psi_{a}(x)$ for $A, B \in M_{2}$, then

(i) $(\lambda A)_{a} \equiv \lambda \phi_{a}(x)$,

(ii) $\left(A^{*}\right)_{a} \equiv \bar{\phi}_{a-1}^{-1}(x a)$,

(iii) $(A+B)_{a} \equiv \phi_{a}(x)+\psi_{a}(x)$,

(iv) $(A B)_{a} \equiv \Sigma_{b \in \varrho} \phi_{b-1}(x) \psi_{b a} \cdot\left(x b^{-1}\right)$. 
Lemma 2.2. If $F_{0} \in H$ is the function where $F_{0}(a, x)=0$ for $a \neq e$ and $F_{0}(e, x)=1$, then $F_{0}$ is a separating cyclic vector for $M_{2}$. For $(A)_{a} \equiv \phi_{a}(x)$,

$$
\left\|A F_{0}\right\|=\left(\sum_{a \in \mathcal{\complement}} \int_{X}\left|\phi_{a}(x a)\right|^{2} d \mu\right) .
$$

Lemma 2.3. Let $U$ be an operator which satisfies $\left\|\left[U, \bar{U}_{g_{i}}\right] F_{0}\right\| \leq \epsilon$ for $i=$ 1,2 and $\epsilon>0$. Then

(i) $\left|\left(U F_{0}, F_{0}\right)\right|>1-K \epsilon$ if $U$ is unitary,

(ii) $\left\|\left(U-\left(U F_{0}, F_{0}\right) I\right) F_{0}\right\|^{2} \leq K^{2} \|\left.\left[U, \bar{U}_{B_{i}}\right] F_{0}\right|^{2}(i=1,2)$ where $K$ is a constant independent of $\epsilon$.

Proof. (i) [10]. This is included in the proof of Lemma 13.

(ii) Letting $(U)_{a} \equiv \phi_{a}(x)$, we note that it suffices to prove the lemma for $U^{*}$. Also, we assume $\left\|\left[U^{*}, \bar{U}_{g_{i}}\right] F_{0}\right\|=\epsilon$. Now

$$
\epsilon^{2}=\left\|\left[U^{*}, \bar{U}_{B_{i}}\right] F_{0}\right\|^{2}=\sum_{a \in \varrho} \int_{X}\left|\phi_{B_{i} a g_{i}^{-1}}\left(x g_{i}^{-1}\right)-\phi_{a}(x)\right|^{2} d \mu
$$

so, by [10, Lemma 13], there is a constant $k$ with

$$
\sum_{a \in \mathfrak{S} ; a \neq(0, e)} \int\left|\phi_{a}(x)\right|^{2} d \mu<k \epsilon^{2}
$$

and a constant $k^{\prime}$ with

$$
\int\left|\phi_{e}(x)\right|^{2} d \mu-\left|\int \phi_{e}(x) d \mu\right|^{2}<k^{\prime} \epsilon^{2}
$$

Now

$$
\begin{aligned}
& \left\|\left(U^{*}-\left(U^{*} F_{0}, F_{0}\right) I\right) F_{0}\right\|^{2}=\left\|U^{*} F_{0}\right\|^{2}-\left|\left(U^{*} F_{0}, F_{0}\right)\right|^{2} \\
& =\left(\sum_{a \in \mathcal{S}} \int_{X}\left|\phi_{a}(x)\right|^{2} d \mu\right)-\left|\int_{X} \phi_{e}(x) d \mu\right|^{2} \\
& =\left(\sum_{a \in \mathfrak{S} ; a \neq(0, e)} \int_{X}\left|\phi_{a}(x)\right|^{2} d \mu\right)+\left(\int_{X}\left|\phi_{e}(x)\right|^{2} d \mu-\left|\int_{X} \phi_{e}(x) d \mu\right|^{2}\right) \\
& <\left(k+k^{\prime}\right) \epsilon^{2}=K^{2}\left\|\left[U^{*}, \bar{U}_{g_{i}}\right] F_{0}\right\|^{2} \text { where } K=\left(k+k^{\prime}\right)^{1 / 2} .
\end{aligned}
$$

Lemma 2.4. Let $\left(U_{n}\right)$ be a central sequence of unitaries in $M_{2}$. Then there is a subsequence (again called $\left(U_{n}\right)$ ) and a scalar $\lambda$ such that $|\lambda|=1$ and strong limit $U_{n}=\lambda I$. 
Proof. Since the unit sphere of a $W^{*}$-algebra is weakly compact, there is a subsequence (called $\left(U_{n}\right)$ ) and an operator $U \in M_{2}$ such that weak limit $U_{n}=U$. Thus weak limit $\left[U_{n}, A\right]=[U, A]$ for each $A \in M_{2}$. But $U_{n}$ is central, so strong limit $\left[U_{n}, A\right]=0$. Thus $[U, A]=0$. Since $M$ is a factor, this means $U=$ $\lambda I$ for some scalar $\lambda$.

According to Lemma 2.3 , if $U_{n}$ is unitary and satisfies $\left\|\left[U_{n}, \bar{U}_{\boldsymbol{B}_{i}}\right] F_{0}\right\|<\epsilon$, $i=1,2$, then $\left|\left(U_{n} F_{0}, F_{0}\right)\right|>1-K_{\epsilon}$, where $K$ is a constant independent of $\epsilon$. Since limit $\left(U_{n} F_{0}, F_{0}\right)=\left(\lambda F_{0}, F_{0}\right)=\lambda$, we have $|\lambda| \geq 1$. But the $U_{n}$ are unitary, so $|\lambda| \leq 1$, i.e. $|\lambda|=1$. Since $U_{n}$ is a sequence of unitaries which converge weakly to the unitary $\lambda l$, the convergence is strong.

Theorem 2.5. $M_{2}$ is not asymptotically abelian.

Proof. Suppose $\left(\rho_{n}\right)$ is a sequence of *automorphisms on $M_{2}$ such that strong limit $\left[\rho_{n}(A), B\right]=0$ for all $A, B \in M_{2}$. Let $E \neq l$ be a nonzero projection in $M_{2}$ and let $F=I-E$. Since any two nonzero projections in $M_{2}$ are equivalent there is a partial isometry $V$ in $M_{2}$ such that $E=V V^{*}$ and $F=V^{*} V . E, F, V$ and $V^{*}$ are a set of matrix units for a $I_{2}$ factor $\mathbb{Q}$. Take $\left(V_{m}\right)$ a finite sequence of unitaries, closed under multiplication and taking adjoints, which generate $\mathfrak{Q}$. For each $V_{m},\left(\rho_{n}\left(V_{m}\right)\right)$ is a central sequence of unitaries in $M_{2}$. Thus, we can find a subsequence (called again $\left(\rho_{n}\right)$ ) of *automorphisms and a set of scalars, $\left(\lambda_{m}\right)$, with $\left|\lambda_{m}\right|=1$ such that strong limit $\rho_{n}\left(V_{m}\right)=\lambda_{m} l$ for each $m$. This defines a function $\phi$ on $\mathcal{Q}$ by the rule that if $A \in \mathbb{Q}$ and $A=\sum_{m=1}^{N} a_{m} V_{m}$, then $\phi(A)=$ $\sum_{m=1}^{N} a_{m} \lambda_{m} \cdot \phi$ is linear and multiplicative. But this is impossible in a $\mathrm{I}_{2}$ factor.

3. Property L. The fact that $M_{2}$ is not asymptotically abelian is consistent with what one might guess. To see this, consider the following definitions.

Definition 3.1. Let $M$ be a factor. $M$ is said to have property $L$ if there is a central sequence $\left(U_{n}\right)$ of unitary operators in $M$ such that weak limit $U_{n}=0$.

Definition 3.2. Let $M$ be a factor. Then $M$ is called asymptotically abelian with invariant state if there is a sequence, $\left(\rho_{n}\right)$, of *-automorphisms on $M$ and a faithful, normal state, $\phi$, on $M$ such that

(i) $\rho_{n}(A)$ is a central sequence for $A \in M$.

(ii) $\phi\left(\rho_{n}(A)\right)=\phi(A)$ for $A \in M$.

Numerous examples exist of infinite factors both with and without preperty L. In particular, Powers' factors, $M_{p^{\prime}} 0<p<1 / 2$, have property $L$ and Pukánszky's $M_{2}$ does not have property $L[10]$. Also, Willig [15] proved that the $I_{\infty}$ factor does not have property $\mathrm{L}$. In type $\mathrm{II}_{1}$ factors, classification has been limited to the study of property $\Gamma$. But property $\Gamma$ is equivalent to property $L$ [16]. Thus the reader can find $\mathrm{II}_{1}$ factors with and without property $L$ by consulting the papers [3] and [4] as well as the standard texts [2], [12], and [13]. We will discuss these cases in the next section. 
It is obvious that if a factor is asymptotically abelian with invariant state, then it is asymptotically abelian. Also, if $M$ is a finite factor, since we can take the trace as $\phi$, asymptotic abelianness with invariant state and asymptotic abelianness are equivalent properties.

Proposition 3.4 will relate Definitions 3.1 and 3.2. First, we need

Lemma 3.3. Let $M$ be a factor which is either continuous or infinite and let $\phi$ be a faithful normal state on $M$. Then, there is a unitary, $U \in M$, such that $\phi(U)=0$.

Proof. Let $A$ be the positive hermitian operator defined by $A x=1 / 2 x$ for $x$ $\epsilon H$ (where $H$ is the underlying Hilbert space for $M$ ). Then $\phi(A)=1 / 2 \phi(I)=1 / 2$. Thus, according to a result of Dye [5], there is a projection, $P \in M$ such that $\phi(P)=1 / 2$. Let $U=(I-P)-P$. Then $U$ is unitary and $\phi(U)=0$.

Proposition 3.4. Let $M$ be a factor with $\operatorname{dim}(M)>1$. If $M$ is asymptotically abelian with invariant state, then $M$ bas property $\mathrm{L}$.

Proof. Let $U$ be a unitary element in $M$ such that $\phi(U)=0$. (If $M$ is a discrete finite factor, we may assume that the invariant state, $\phi$, is the normalized trace.) Define $\left(U_{n}\right)$ by $U_{n}=\rho_{n}(U)$. This gives a sequence of unitaries such that strong limit $\left[U_{n}, A\right]=0$ for all $A \in M$. We need only show that weak $\operatorname{limit} U_{n}$ $=0$.

The sequence $\left(U_{n}\right)$ is uniformly bounded so, by weak compactness, there is a subsequence (again called $\left(U_{n}\right)$ ) and a $U_{0} \in M$ such that weak limit $U_{n}=U_{0}$. Then weak limit $\left[U_{n^{\prime}} A\right]=\left[U_{0}, A\right]$ for each $A \in M$. Since strong $\operatorname{limit}\left[U_{n^{\prime}} A\right]=0$, we have $\left[U_{0}, A\right]=0$ for all $A \in M$. This means $U_{0}=\lambda I$ for some scalar $\lambda$.

We know $\phi\left(U_{n}\right)=\phi\left(\rho_{n}(U)\right)=\phi(U)=0$. But $\phi$ is weakly continuous. This means limit $\phi\left(U_{n}\right)=\phi(\lambda I)=\lambda$. Combining, we have $\lambda=0$ or weak limit $U_{n}=0$.

Corollary 3.5. Let $M$ be a finite factor, with $\operatorname{dim}(M)>1$. If $M$ is asymptotically abelian, then $M$ has property $\mathrm{L}$.

Proof. This is an immediate consequence of the equivalence of asymptotic abelianness with invariant state and asymptotic abelianness in finite factors.

We may now conclude that any factor which does not have property $L$ is not asymptotically abelian with invariant state and a finite factor without property $\mathrm{L}$ is not asymptotically abelian (if $\operatorname{dim}(M)>1$ ). It is probably also true, though it has not yet been proven, that infinite factors without property $L$ are not asymptotically abelian. The converse is false in the $\mathrm{II}_{1}$ case because the factor $\mathrm{U}\left(\Phi_{2} \times \Pi\right)$, where $\Phi_{2}$ is the free group on two generators and $\Pi$ is the group of all finite permutations on the integers, satisfies property $L$ but is not asymptotically abelian. (We will show in the next section that the converse is also false in the $\mathrm{II}_{\infty}$ case.) 
Pukánszky [10] has shown that $M_{2}$ does not have property L. Using Proposition 3.4 we can immediately conclude that $M_{2}$ is not asymptotically abelian with invariant state and tentatively guess that it is not asymptotically abelian. Theorem 2.5 proved that $M_{2}$, indeed, is not asymptotically abelian.

If we carry this line of thought further, we may also guess that if there is an asymptotically abelian infinite factor, it would probably be a factor that has property L. Also, we will see in the next section that it must be a type III factor. Powers' factors, $M_{p}, 0<p<1 / 2$, satisfy both these conditions and thus are the most likely candidates. Conversely, if Powers' factors are not asymptotically abelian, it is reasonable to assume that no other infinite factor is asymptotically abelian.

4. Factors of type $\mathrm{II}_{\infty}$. In this section we will prove that the $\mathrm{II}_{\infty}$ factors are not asymptotically abelian. Thus, there are infinite factors which satisfy property $\mathrm{L}$ but which are not asymptotically abelian. Also, we can conclude that if there are any asymptotically abelian infinite factors, they must be type III.

Each factor of type $\mathrm{II}_{\infty}$ is spatially isomorphic to a factor $M \otimes B(\mathcal{H})$ where $M$ is a factor of type $\mathrm{II}_{1}$ and $B(\mathcal{H})$ is the $\mathrm{I}_{\infty}$ factor. We will therefore identify our type $\mathrm{II}_{\infty}$ factors with $M \otimes B(\mathcal{H})$ and use this notation. The other notation used in this section is as follows: $K$ will be the underlying Hilbert space for $M$ and $\mathcal{H}$, is the Hilbert space for $B(\mathcal{H}) ;\left\{e_{i}\right\}_{i=1}^{\infty}$ denotes an orthonormal basis of $\mathcal{H}$, and $\left\{E_{i j}\right\}_{i, j=1}^{\infty}$ is the corresponding basis for $B(\mathcal{H})$. An operator, $A$, in $M \otimes B(\mathcal{H})$ can be written as an infinite matrix $\left(A_{i j}\right)$ whose entries are elements of $M$. For matrices with a finite number of nonzero entries, we will use the tensor product notation, i.e. $X \otimes E_{i j}$ is the matrix with $X$ in the $i, j$-entry and zeroes elsewhere.

Definition 4.1. Let $A=\left(A_{i j}\right)$ be an operator in $M \otimes B(\mathcal{H})$. If $A_{i j}=0$ for $i \neq j$, then $A$ will be called a diagonal operator.

Lemma 4.2. Let $M$ be a $W^{*}$-algebra and $\left(A_{n}\right)$ a central sequence in $M \otimes B(\mathcal{H})$. Then there is a central sequence of diagonal operators $\left(B_{n}\right)$ in $M \otimes B(\mathcal{H})$ which satisfy $\left\|B_{n}\right\| \leq\left\|A_{n}\right\|$ and such that strong limit $\left(A_{n}-B_{n}\right)=0$.

Proof. Let $A_{n}=\left(A_{i j}^{(n)}\right)$ and define $B_{n}=\left(\delta_{i j} A_{i j}^{(n)}\right)$. Clearly $B_{n}$ is diagonal for each $n$. Take $X_{m}=I \otimes E_{m m} \in M \otimes B(\mathcal{H})$ where $I$ is the unit in $M$ and take $x_{m}=f \otimes e_{m} \in \mathcal{K} \otimes \mathcal{H}$ where $f$ is an arbitrary element of $\mathcal{K}$. Then it suffices to prove that limit $\left\|\left(A_{n}-B_{n}\right) x_{m}\right\|=0$ for all $m$.

We first note that $X_{m} x_{m}=x_{m}$ and $A_{n} x_{m}=\Sigma_{i=1}^{\infty} A_{i m}^{(n)} f \otimes e_{i}$. Then, using the fact that $A_{n}$ is central, we have, for large $n$ and for all $m$,

$$
\epsilon>\left\|\left(\Lambda_{n} X_{m}-X_{m} \Lambda_{n}\right) x_{m}\right\|=\left\|\left(\sum_{i=1}^{\infty} A_{i m}^{(n)} f \otimes e_{i}\right)-\left(\Lambda_{m m}^{(n)} f \otimes e_{m}\right)\right\| \text {. }
$$


Thus, for large $n$ and all $m$,

$$
\left\|\left(A_{n}-B_{n}\right) x_{m}\right\|=\left\|\left(\sum_{i=1}^{\infty} A_{i m}^{(n)} f \otimes e_{i}\right)-\left(A_{m m}^{(n)} f \otimes e_{m}\right)\right\|<\epsilon .
$$

To show the boundedness property, we note that $\left\|B_{n}\right\|=\sup _{m}\left\|A_{m m}^{(n)}\right\|$. Choose $x_{m}$ as above so that $\|f\|=1$. Then $\sup _{m}\left\|A_{n} x_{m}\right\| \leq\left\|A_{n}\right\|$. But,

$$
\left\|A_{n} x_{m}\right\|^{2}=\left\|\sum_{i=1}^{\infty} A_{i m}^{(n)} f \otimes e_{i}\right\|^{2}=\sum_{i=1}^{\infty}\left\|A_{i m}^{(n)} f\right\|^{2} \geq\left\|A_{m m}^{(n)} f\right\|^{2} .
$$

So, sup $\left\|A_{m m}^{(n)} f\right\| \leq\left\|A_{n}\right\|$ and since $\|f\|=1$ is arbitrary

$$
\left\|B_{n}\right\|=\sup _{m}\left(\sup _{\|f\|=1}\left\|A_{m m}^{(n)} f\right\|\right) \leq\left\|A_{n}\right\| .
$$

Lemma 4.3. If $\left(B_{n}\right)$ is a central sequence of diagonal operators as in Lemma 4.2 , there is a sequence $\left(F_{n}\right)$ in $M \otimes\{I\}$ satisfying $\left\|F_{n}\right\| \leq\left\|B_{n}\right\|$ for each $n$ and such that strong limit $\left(B_{n}-F_{n}\right)=0$.

Proof. Let $\left(B_{n}\right), f$, and $x_{m}$ be as in Lemma 4.2. Define $Y_{m}=I \otimes\left(E_{1 m}+\right.$ $\left.E_{m 1}\right) \in M \otimes B(\mathcal{H})$ and $y_{m}=f \otimes\left(e_{1}+e_{m}\right) \in \mathcal{K} \otimes \mathcal{H}$. We assume $m \neq 1$. Then $Y_{m} y_{m}$ $=y_{m}, Y_{m} x_{m}=x_{1}, Y_{m} x_{1}=x_{m}$ and $B_{n} y_{m}=A_{11}^{(n)} f \otimes e_{1}+A_{m m}^{(n)} f \otimes e_{m}$. So, for large $n$ and an arbitrary $m \neq 1$, we have

$$
\begin{aligned}
\epsilon & >\left\|\left(B_{n} Y_{m}-Y_{m} B_{n}\right) y_{m}\right\| \\
& =\left\|\left(A_{11}^{(n)} f \otimes e_{1}+A_{m m}^{(n)} f \otimes e_{m}\right)-\left(A_{11}^{(n)} f \otimes e_{m}+A_{m m}^{(n)} f \otimes e_{1}\right)\right\| \\
& =\left\|\left(A_{11}^{(n)}-A_{m m}^{(n)}\right) f \otimes\left(e_{1}-e_{m}\right)\right\|=\sqrt{2}\left\|A_{11}^{(n)} f-A_{m m}^{(n)} f\right\| .
\end{aligned}
$$

Now, defining $F_{n}=A_{11}^{(n)} \otimes I \in M \otimes\{I\}$, we have

$$
\begin{aligned}
\left\|\left(B_{n}-F_{n}\right) x_{m}\right\| & =\left\|\left(A_{m m}^{(n)} f \otimes e_{m}\right)-\left(A_{11}^{(n)} f \otimes e_{m}\right)\right\| \\
& =\left\|A_{11}^{(n)} f-A_{m m}^{(n)} f\right\|<\epsilon / \sqrt{2}
\end{aligned}
$$

which proves strong limit $\left(B_{n}-F_{n}\right)=0$.

The boundedness is easy to verify since

$$
\begin{aligned}
\left\|F_{n}\right\| & =\sup _{\|x\|=1}\left\|\left(A_{11}^{(n)} \otimes I\right) x\right\|=\sup _{\|f\|=1}\left\|A_{11}^{(n)} f\right\| \\
& \leq \sup _{\|f\|=1}\left(\sup _{i}\left\|A_{i i}^{(n)} f\right\|\right) \leq\left\|B_{n}\right\| .
\end{aligned}
$$


Thus, any central sequence in $M \otimes B(\mathcal{H})$ is equivalent to a sequence in $M$. This enables us to prove.

Theorem 4.4. If $M$ is a $\mathrm{II}_{1}$ factor, then $M \otimes B(\mathcal{H})$ is not asymptotically abelian.

Proof. Suppose $M \otimes B(\mathcal{H})$ were asymptotically abelian. Let $\left(\rho_{n}\right)$ be a sequence *-automorphisms of $M \otimes B(\mathcal{H})$ such that $\left(\rho_{n}(A)\right)$ is central for all $A$ in $M \otimes B(\mathcal{H})$. Choose $A_{1}$ and $A_{2}$, arbitrary elements of $M \otimes B(\mathcal{H})$. Then $\left(\rho_{n}\left(A_{1}\right)\right)$ and $\left(\rho_{n}\left(A_{2}\right)\right)$ are central sequences. Thus, combining the results of Lemma 4.2 and Lemma 4.3 , we can find sequences, $\left(F_{n}^{(1)}\right)$ and $\left(F_{n}^{(2)}\right)$, in $M$ which satisfy strong $\operatorname{limit}\left(\rho_{n}\left(A_{i}\right)-F_{n}^{(i)}\right)=0$ and strong $\operatorname{limit}\left(\rho_{n}\left(A_{i}\right)^{*}-F_{n}^{(i) *}\right)=0$ for $i=1,2$. This means that for $x \in \mathcal{K} \otimes \mathcal{H}$ and $n$ large,

$$
\begin{aligned}
\mid\left(\left(\rho_{n}\left(A_{1} A_{2}\right)\right.\right. & \left.\left.-F_{n}^{(1)} F_{n}^{(2)}\right) x, x\right) \mid \\
& =\left|\left(\rho_{n}\left(A_{2}\right) x, \rho_{n}\left(A_{1}\right) * x\right)-\left(F_{n}^{(2)} x, \underset{n}{(1) *} x\right)\right| \\
& =\left|\left(\left(\rho_{n}\left(A_{2}\right)-F_{n}^{(2)}\right) x, \rho_{n}\left(A_{1}\right)^{*} x\right)+\left(F_{n}^{(2)} x,\left(\rho_{n}\left(A_{1}\right)^{*}-F_{n}^{(1) *}\right) x\right)\right| \\
& \left.\leq\left\|\left(\rho_{n}\left(A_{2}\right)-F_{n}^{(2)}\right) x\right\|\left\|\rho_{n}\left(A_{1}\right)^{*} x\right\|+\left\|F_{n}^{(2)} x\right\| \|\left(\rho_{n}\left(A_{1}\right)^{*}-F_{n}^{(1) *}\right) x\right) \| \\
& \leq \epsilon \rho_{n}\left(A_{1}\right)\|+\| F_{n}^{(2)}\|\epsilon \leq \epsilon\| \rho_{n}\left(A_{1}\right)\|+\| \rho_{n}\left(A_{2}\right) \| \epsilon \\
& =\epsilon\left\|A_{1}\right\|+\left\|A_{2}\right\| \epsilon=\left(\left\|A_{1}\right\|+\left\|A_{2}\right\|\right) \epsilon .
\end{aligned}
$$

Thus, weak limit $\left(\rho_{n}\left(A_{1} A_{2}\right)-F_{n}^{(1)} F_{n}^{(2)}\right)=0$. The same reasoning will show that weak $\operatorname{limit}\left(\rho_{n}\left(A_{2} A_{1}\right)-F_{n}^{(2)} F_{n}^{(1)}\right)=0$.

Now let $\psi$ be a normal state on $B(\mathcal{H})$ and tr the trace on $M$. Then $\phi_{0}=$ $\operatorname{tr} \otimes \psi$ is a normal state on $M \otimes B(\mathcal{H})$. This means, since $\phi_{0}$ is weakly continuous on bounded sets, that

$$
\left|\phi_{0}\left(\rho_{n}\left(A_{1} A_{2}\right)-F_{n}^{(1)} F_{n}^{(2)}\right)\right|<\epsilon, \quad\left|\phi_{0}\left(\rho_{n}\left(A_{2} A_{1}\right)-F_{n}^{(2)} F_{n}^{(1)}\right)\right|<\epsilon
$$

for large $n$. Also, since $\phi_{0}$ is the trace when restricted to $M$, we have $\phi_{0}\left(F_{n}^{(1)} F_{n}^{(2)}\right)$ $=\phi_{0}\left(F_{n}^{(2)} F_{n}^{(1)}\right)$.

Define a sequence $\left(\phi_{n}\right)$ of normal states on $M \otimes B(\mathcal{H})$ by $\phi_{n}(A)=\phi_{0}\left(\rho_{n}(A)\right)$. Then, by weak *-compactness of bounded sets in the dual, $\left(\phi_{n}\right)$ has an accumulation point, $\bar{\phi}$. Thus, for arbitrarily large $n_{0}$, there exists a positive integer $n$ such that $n>n_{0}$ and

$$
\left|\bar{\phi}\left(A_{1} A_{2}\right)-\phi_{0}\left(\rho_{n}\left(A_{1} A_{2}\right)\right)\right|<\epsilon, \quad\left|\bar{\phi}\left(A_{2} A_{1}\right)-\phi_{0}\left(\rho_{n}\left(A_{2} A_{1}\right)\right)\right|<\epsilon .
$$


Thus $\bar{\phi}$ is a state on $M \otimes B(\mathcal{H})$ which satisfies $\bar{\phi}\left(A_{1} A_{2}\right)=\bar{\phi}\left(A_{2} A_{1}\right)$ for all $A_{1}$, $A_{2} \in M \otimes B(\mathcal{H})$. But $M \otimes B(\mathcal{H})$ is infinite. Hence, there is a nonzero projection, $e$, such that $e \sim I-e \sim I$. Let $V_{1}$ and $V_{2}$ be partial isometries in $M \otimes B(\mathcal{H})$ such that $V_{1} V_{1}^{*}=e$ and $V_{2} V_{2}^{*}=I-e$ and $V_{1}^{*} V_{1}=V_{2}^{*} V_{2}=I$. Then

$\bar{\phi}(e)=\bar{\phi}\left(V_{1} V_{1}^{*}\right)=\bar{\phi}\left(V_{1}^{*} V_{1}\right)=\bar{\phi}(I)=1, \quad \bar{\phi}(I-e)=\bar{\phi}\left(V_{2} V_{2}^{*}\right)=\bar{\phi}\left(V_{2}^{*} V_{2}\right)=\bar{\phi}(I)=1$.

Thus, $\bar{\phi}(I)=\bar{\phi}(I-e)+\bar{\phi}(e)=2$ which is a contradiction.

Using this theorem, we can exhibit infinite factors which satisfy property $L$, but are not asymptotically abelian. Consider first, the following result of Tomiyama [14].

Theorem 4.5. Let $M$ and $N$ be factors and suppose that $M$ or $N$ bas property L. Then $M \otimes N$ bas property $\mathrm{L}$.

Now, let $M$ be any $\mathrm{II}_{1}$ factor with property L. An example would be the hyperfinite factor $M=\mathcal{U}(\mathrm{II})$ where $\Pi$ is the group of permutations on the integers. (For other examples, see those factors in [3] and [4] which have property $\Gamma$.) Then $\mathcal{U}(\Pi) \otimes B(\mathcal{H})$ is an infinite factor with property $\mathrm{L}$, but $\mathrm{U}(\Pi) \otimes B(\mathcal{H})$, being $\mathrm{II}_{\infty}$, is not asymptotically abelian.

One other interesting result of Lemmas 4.2 and 4.3 is that every central sequence in $B(\mathcal{H})$ is trivial. This is easy to see if we let $M$ be the $I_{1}$ factor. Then, every central sequence in $M \otimes B(\mathcal{H})$ is equivalent to a sequence in $M$ which is, in this case, a sequence of scalars.

5. Central sequences in tensor products. Lemmas 4.2 and 4.3 led to the conclusion that any central sequence in $M \otimes B(\mathcal{H})$ is equivalent to a sequence in $M$. We now look at another case where a central sequence in a tensor product is equivalent to a sequence in one of the tensored factors, namely the algebra $M_{2}$ $\otimes N$ where $M_{2}$ is the type III factor constructed in $\$ 2$ and $N$ is a II $_{1}$ factor such that $N=\mathcal{U}(G)$ for some group, $G$, with infinite conjugate classes.

First, we point out that $M_{2} \otimes N$ has $L_{\mu}^{2}(X) \otimes l^{2}(G)$ as its underlying Hilbert space with $F_{0} \otimes \delta_{e}$ a separating cyclic vector. We will use the notation from the paper of Ching [1] to prove our theorem.

An element $F \otimes f$ of $L_{\mu}^{2}(X) \otimes l^{2}(G)$ will be denoted $(f(g) F)$ i.e. $(F \otimes f)_{g}=$ $f(g) F$. A sequence of operators $\left(W_{n}\right) \subset M_{2} \otimes N$ will be of the form $W_{n}=\left(W_{g}^{(n)}\right)_{g \in G}$ where $W_{\mathcal{B}}^{(n)} \in M_{2}$ for each $g \in G$ and $\left(W_{8}^{(n)}\right)_{a} \equiv \phi_{a, 8}^{(n)}(x)$ for $a \in \mathcal{G}$. Also, $W_{n}$ acts on $L_{\mu}^{2}(X) \otimes l^{2}(G)$ in the following manner

$$
\left(W_{n}(F \otimes f)\right)_{g}=\sum_{b \in G} W_{b}^{(n)} f(g b) F .
$$

Theorem 5.1. If $\left(W_{n}\right)$ is a central sequence in $M_{2} \otimes N$, there is a sequence $\left(f_{n}\right)$ in $N$ such that strong limit $\left(W_{n}-I \otimes f_{n}\right)=0$. 
Proof. For $n$ fixed, let the function $f_{n}: G \rightarrow \mathrm{C}$ be defined by $f_{n}(g)=$ $\int_{X} \phi_{e, g^{-1}}^{(n)}(x) d \mu=\left(W_{g^{-1}}^{(n)} F_{0}, F_{0}\right)$. Note that

$$
\left(W_{n}\left(F_{0} \otimes \delta_{e}\right)\right)_{g}=\sum_{b \in G} W_{b}^{(n)} \delta_{e}(g b) F_{0}=W_{g^{-1}}^{(n)} F_{0}
$$

so

$$
\begin{aligned}
& \left\|\left(W_{n}-I \otimes f_{n}\right)\left(F_{0} \otimes \delta_{e}\right)\right\|^{2} \\
& \quad=\left\|\left(W_{g^{-1}}^{(n)} F_{0}-\left(W_{g^{-1}}^{(n)} F_{0}, F_{0}\right) F_{0}\right)_{g \in G}\right\|^{2}=\sum_{g^{\epsilon G}}\left\|\left(W_{g}^{(n)}-\left(W_{g}^{(n)} F_{0}, F_{0}\right) I\right) F_{0}\right\|^{2} .
\end{aligned}
$$

Now

$$
\begin{gathered}
\left(W_{n}\left(\bar{U}_{g_{i}} \otimes \delta_{e}\right)\left(F_{0} \otimes \delta_{e}\right)\right)_{\boldsymbol{g}}=\left(W_{n}\left(\bar{U}_{g_{i}} F_{0} \otimes \delta_{e}\right)\right)_{g} \\
=\sum_{b \in G} W_{b}^{(n)} \delta_{e}(g b) \bar{U}_{g_{i}} F_{0}=W_{\boldsymbol{g}^{-1}}^{(n)} \bar{U}_{g_{i}} F_{0}
\end{gathered}
$$

and

$$
\begin{aligned}
\left(\left(\bar{U}_{g_{i}}\right.\right. & \left.\left.\otimes \delta_{e}\right) W_{n}\left(F_{0} \otimes \delta_{e}\right)\right)_{g}=\left(\left(\bar{U}_{g_{i}} \otimes \delta_{e}\right)\left(\left(W_{k}^{(n)} F_{0}\right)\right)\right)_{g} \\
& =\left(\left(\delta_{e}(k) \bar{U}_{g_{i}}\right)_{k \in G}\left(\left(W_{k-1}^{(n)} F_{0}\right)\right)\right)_{g}=\sum_{b \in G} \delta_{e}(b) \bar{U}_{g_{i}} W_{(g b)-1}^{(n)} F_{0}=\bar{U}_{g_{i}} W_{g^{-1}}^{(n)} F_{0} .
\end{aligned}
$$

Hence, using the fact that $\left(W_{n}\right)$ is a central sequence, given $\epsilon>0$, we can find $n$ such that

$$
\begin{aligned}
& \epsilon>\left\|\left[W_{n}, \bar{U}_{g_{i}} \otimes \delta_{e}\right]\left(F_{0} \otimes \delta_{e}\right)\right\|^{2} \\
& \left.=\| \underset{g^{-1}}{(n)} \bar{U}_{g_{i}} F_{0}-\bar{U}_{g_{i}{ }^{-1}} W^{(n)} F_{0}\right)\left\|^{2}=\sum_{g \in G}\right\|\left[W_{g^{-1}}^{(n)}, \bar{U}_{g_{i}}\right] F_{0} \|^{2} .
\end{aligned}
$$

Now, using Lemma 2.3,

$$
\left\|\left(W_{g}^{(n)}-\left(W_{g}^{(n)} F_{0}, F_{0}\right) l\right) F_{0}\right\|^{2}<K\left\|\left[W_{g}^{(n)}, \bar{U}_{g_{i}}\right] F_{0}\right\|^{2}
$$

so that

$$
\begin{aligned}
& \text { limit }\left\|\left(W_{n}-\left(I \otimes f_{n}\right)\right)\left(F_{0} \otimes \delta_{e}\right)\right\|^{2} \\
&= \operatorname{limit} \sum_{g^{\epsilon G}}\left\|\left(W_{g}^{(n)}-\left(W_{g}^{(n)} F_{0}, F_{0}\right) I\right) F_{0}\right\|^{2} \\
& \leq K \operatorname{limit} \sum_{g^{\in G}}\left\|\left[W_{g}^{(n)}, \bar{U}_{g_{i}}\right] F_{0}\right\|^{2} \\
&=K \operatorname{limit}\left\|\left[W_{n}, \bar{U}_{g_{i}} \otimes \delta_{e}\right]\left(F_{0} \otimes \delta_{e}\right)\right\|^{2}=0 .
\end{aligned}
$$


It remains to show that $f_{n} \in \mathrm{U}(G)$ for all $n$. This means, given $f \in l^{2}(G)$, we must show $\left\|f * f_{n}\right\|_{2} \leq K\|f\|_{2}$ where $\|\cdot\|_{2}$ is the trace norm. We may assume, without loss of generality, that $\left\|W_{n}\right\| \leq 1$ and $K=1$. Take $X$ and $Y$ in $L_{\mu}^{2}(X)$ $\otimes l^{2}(G)$ defined by $X=F_{0} \otimes f$ and $Y=F_{0} \otimes f * f_{n}$. We know that $\left(W_{n} X, Y\right) \leq$ $\left\|W_{n} X\right\|\|Y\| \leq\|X\|\|Y\|$. Computing, we get

$$
\|X\|=\left\|F_{0} \otimes f\right\|=\|f\|_{2}, \quad\|Y\|=\left\|F_{0} \otimes f * f_{n}\right\|=\left\|f * f_{n}\right\|_{2}
$$

and

so that

$$
\left(W_{n} X\right)_{8}=\left(\left(W_{b}^{(n)}\right)_{b \in G}\left(F_{0} \otimes f\right)\right)_{8}=\sum_{b \in G} W_{b}^{(n)} f(g h) F_{0}
$$

$$
\begin{aligned}
\left(W_{n} X, Y\right) & =\sum_{g^{\in G}}\left(\sum_{b \in G} W_{b}^{(n)} f(g b) F_{0},\left(f * f_{n}\right)(g) F_{0}\right) \\
& =\sum_{g^{\in G}} \overline{\left(\left(f * f_{n}\right)(g)\right)}\left(\sum_{b \in G} W_{b}^{(n)} f(g b) F_{0}, F_{0}\right) \\
& =\sum_{g^{\in G G}} \overline{\left(f * f_{n}\right)(g)}\left(\sum_{b \in G} f(g b)\left(W_{b}^{(n)} F_{0}, F_{0}\right)\right) \\
& =\sum_{g^{\in G}} \overline{\left(f * f_{n}\right)(g)} \sum_{b \in G} f(g b) f_{n}\left(b^{-1}\right) \\
& =\sum_{g^{\in G}} \overline{\left(f * f_{n}\right)(g)}\left(f * f_{n}\right)(g)=\left\|f * f_{n}\right\|_{2}^{2} .
\end{aligned}
$$

Combining gives

$$
\left\|f * f_{n}\right\|_{2}^{2} \leq\|f\|_{2}\left\|f * f_{n}\right\|_{2} \text { or }\left\|f * f_{n}\right\|_{2} \leq\|f\|_{2} .
$$

Thus, a central sequence in $M_{2} \otimes N$ is equivalent to a sequence in $N$ and we may reduce any discussion of central sequences in $M_{2} \otimes N$ to a discussion of sequences in $N$. (Just as in the previous section we were able to consider a sequence in $M$ in place of a sequence in $M \otimes B(\mathcal{H})$.) Does the opposite work? That is, may we extend a central sequence in $N$ to a central sequence in $M_{2} \otimes$ $N$. The answer is "yes" and is covered by the following.

Lemma 5.2. Let $M$ be a finite $W^{*}$-algebra. Define $C_{M}^{0}=\left(\bigoplus_{\infty} M / I_{0}\right) \cap\left(\rho(\tilde{M})^{\prime}\right)$, where $\bigoplus_{\infty} M$ is the direct sum of a countable number of copies of $M, I_{0}=\left\{\left(A_{n}\right) \epsilon\right.$ $\left.\bigoplus_{\infty} M \mid\left\|A_{n}\right\|_{2} \rightarrow 0\right\}, \widetilde{M}=\left\{\left(A_{n}\right) \in \bigoplus_{\infty} M \mid A_{n}=A\right.$ for all $\left.n\right\}$ and $\rho$ is the canonical map of $\bigoplus_{\infty} M$ onto $\bigoplus_{\infty} M / I_{0}$. Then $C_{M}^{0}$ is a finite $W^{*}$-algebra.

Proof. $[8, \S 4]$. 
Lemma 5.3. Let $M$ be $a W^{*}$-algebra and $T$ a bermitian element of $M$. If $f$ is a continuous function on the real numbers, then $f(T)$ is in $M$.

Proof. [2, Chapter I, $\$ 1.2]$.

Lemma 5.4. Let $M$ and $N$ be factors. Suppose $\left(U_{n}\right)$ is a sequence of unitaries in $N$ such that strong limit $U_{n}^{*} A U_{n}=A$ for all $A$ in $N$. If $\widetilde{U}_{n \sim}=I \otimes U_{n^{\prime}}$ then $\tilde{U}_{n}$ is a sequence of unitaries in $M \otimes N$ such that strong limit $\tilde{U}_{n}^{*} X \tilde{U}_{n}=X$ for all $X$ in $M: \otimes N$.

Proof. [14]. This is included in the proof of Theorem 2.

Lemma 5.4 answers our question if we assume that the central sequence is a sequence of unitaries. In the next theorem, we will remove this condition (which is essential to Tomiyama's proof). However, in order to do this, we must impose another condition: One of the tensored factors must be semifinite.

Theorem 5.5. Let $N$ be a finite factor and $M$ an arbitrary factor. If $\left(A_{n}\right)$ is a central sequence in $N$, then $\left(I \otimes A_{n}\right)$ is central in $M \otimes N$.

Proof. Our method will be to reduce the $A_{n}$ 's to unitary operators. First,

$$
A_{n}=1 / 2\left(A_{n}+A_{n}^{*}\right)+i\left(1 / 2\left(i A_{n}^{*}-i A_{n}\right)\right)
$$

Since $N$ is a finite factor, $\left(A_{n}\right)$ is a central sequence if and only if $\left(A_{n}^{*}\right)$ is central. Thus, $\left(A_{n}\right)$ is central if and only if both $1 / 2\left(A_{n}+A_{n}^{*}\right)$ and $1 / 2\left(i A_{n}^{*}-i A_{n}\right)$ are central. This means that the theorem is true if and only if it is true for hermitian operators. Assume, therefore, that $A_{n}$ is hermitian.

Lemmas 5.2 and 5.3 imply that if $\left(A_{n}\right)$ is a hermitian central sequence, then $\left(f\left(A_{n}\right)\right)=\left(\left(I-A_{n}^{2}\right)^{1 / 2}\right)$ is a central sequence. Now (we may assume $\left\|A_{n}\right\| \leq 1$ )

$$
A_{n}=1 / 2\left(A_{n}+i\left(I-A_{n}^{2}\right)^{1 / 2}\right)+1 / 2\left(A_{n}-i\left(I-A_{n}^{2}\right)^{1 / 2}\right) .
$$

So, the theorem is true if and only if it is true for unitary operators. But, according to Lemma 5.4, the theorem is true for unitary operators.

I am indebted to P. Willig for suggesting the following.

Theorem 5.6. Let $\tilde{N}$ be a semifinite factor and $M$ an arbitrary factor. If $\left(A_{n}\right)$ is central in $\tilde{N}$, then $\left(I \otimes A_{n}\right)$ is central in $M \otimes \tilde{N}$.

Proof. Let $\tilde{N}=B(\mathcal{H}) \otimes N$ with $N$ a finite factor. Then, using the results of $\$ 4$, there is a central sequence $\left(F_{n}\right)$ in $N$ such that the sequence $\left(I \otimes F_{n}\right)$ is equivalent to $\left(A_{n}\right)$. But $\left(I \otimes A_{n}\right)$ is equivalent to $\left(I \otimes\left(I \otimes F_{n}\right)\right)=\left((I \otimes I) \otimes F_{n}\right)$ which, according to Theorem 5.5, is central in $(M \otimes B(\mathcal{H})) \otimes N=M \otimes \tilde{N}$. Thus' $\left(I \otimes A_{n}\right)$ is central in $M \otimes \tilde{N}$. 
Thus, an arbitrary central sequence in a semifinite factor, $N$, is still a central sequence when extended to the tensor product, $M \otimes N$. Can this be generalized to the case where $N$ is purely infinite? Obviously, if a purely infinite factor, $N$, satisfies the property that every central sequence is trivial, the answer, for $N$, is "yes". In general, however, the answer to this question is not known.

6. Uniform asymptotic abelianness. Finally, we investigate another aspect of the property of asymptotic abelianness. Algebras with asymptotic abelianness in physical situations are $C^{*}$-algebras and not $W^{*}$-algebras. If an algebra, $M$, is a $C^{*}$-algebra, but not a $W^{*}$-algebra, convergent sequences are considered to be those which converge uniformly. Therefore, in discussing those properties of $C^{*}$-algebras which relate to convergence of sequences, one would confine oneself to the uniform topology. It is natural to wonder whether this property would be useful in the case of $W^{*}$-algebras.

We will first formally define the property under consideration.

Definition 6.1. Let $M$ be a $W^{*}$-algebra. $M$ is called uniformly asymptotically abelian if there is a sequence, $\left(\rho_{n}\right)$, of $*$-automorphisms on $M$ such that limit $\left\|\left[\rho_{n}(A), B\right]\right\|=0$ for all $A, B \in M$.

Obviously, any uniformly asymptotically abelian algebra is asymptotically abelian. However, the converse is not the case. This fact will be a trivial consequence of the theorem in this section.

The following is a result of Kallman ([6], [7]).

Lemma 6.2. Let $M$ be a $W^{*}$-algebra on a separable Hilbert space. If $\phi(n)$ is a sequence of *-automorphisms of $M$ such that $\operatorname{limit}_{n \rightarrow \infty}\|\phi(n)(T)-T\|=0$ for all $T \in M$, then

$$
\operatorname{limit}_{n \rightarrow \infty}\left(\sup _{\|T\| \leq 1}\{\|\phi(n)(T)-T\|\}\right)=0 .
$$

We use this lemma to show that $W^{*}$-algebras are not uniformly asymptotically abelian (unless they are commutative algebras).

Theorem 6.3. Let $M$ be a noncommutative $W^{*}$-algebra. $M$ is not uniformly asymptotically abelian.

Proof. Suppose $M$ were uniformly asymptotically abelian. Let $\left(\rho_{n}\right)$ be the sequence of automorphisms of Definition 6.1. Then if $U$ is a unitary operator in $M, \rho_{n}(U)$ is a unitary operator for each $n$ and $\left(\rho_{n}(U)\right)$ is a central sequence in $M$. Thus,

$$
\operatorname{limit}_{n \rightarrow \infty}\left\|\rho_{n}(U)^{*} T \rho_{n}(U)-T\right\|=\operatorname{limit}_{n \rightarrow \infty}\left\|T \rho_{n}(U)-\rho_{n}(U) T\right\|=0 .
$$


Let $\phi$ of Lemma 6.2 be defined by $\phi(n)(T)=\rho_{n}(U)^{*} T \rho_{n}(U)$. Then, using Lemma 6.2 , we have

$$
\operatorname{limit}_{n \rightarrow \infty}\left(\sup _{\|T\| \leq 1}\left\{\left\|\rho_{n}(U)^{*} T \rho_{n}(U)-T\right\|\right\}\right)=0
$$

But

$$
\left\|\rho_{n}(U)^{*} T \rho_{n}(U)-T\right\|=\left\|\rho_{n}\left(U^{*} \rho_{n}^{-1}(T) U-\rho_{n}^{-1}(T)\right)\right\|=\left\|U^{*} \rho_{n}^{-1}(T) U-\rho_{n}^{-1}(T)\right\| .
$$

Thus, given $\epsilon$, there is an $n_{0}$ such that

$$
\sup _{\|T\| \leq 1}\left\|U^{*} \rho_{n_{0}}^{-1}(T) U-\rho_{n_{0}}^{-1}(T)\right\|<\epsilon .
$$

But $\rho_{n_{0}}^{-1}$ is a *-automorphism and, in particular, preserves the unit ball. So, given $\epsilon$,

$$
\sup _{\|T\|<1}\left\|U^{*} T U-T\right\|<\epsilon
$$

i.e. $\left\|U^{*} T U-T\right\|=0$ for all $T \in M$ such that $\|T\| \leq 1$. But this means $[U, T]=0$ for all $T \in M$ which is impossible. Thus, there are no uniformly asymptotically abelian noncommutative $W^{*}$-algebras (even though there are many algebras which are asymptotically abelian) and we can consider this property as one that is special to $C^{*}$-algebras.

\section{BIBLIOGRAPHY}

1. W. Ching, Non-isomorphic, non-hyperfinite fact ors, Canad. J. Math. 21 (1969), 1293-1308. MR 40 \#7822.

2. J. Dixmier, Les algèbres d'opérateurs dans l'espace hilbertien, 2nd ed., GauthierVillars, Paris, 1969.

3. - Quelques propriétés des suits centrales dans les facteurs de type $\mathrm{II}_{1}$, Invent. Math. 7 (1969), 215-225. MR 40 \#1786.

4. J. Dixmier and E. C. Lance, Deux nouveaux factuers de type $\mathrm{II}_{1}$, Invent. Math. 7 (1969), 226-234. MR $40 \# 1787$.

5. H. Dye, The unitary structure in finite rings of operators, Duke Math. J. 20 (1953), 55-69. MR 14, 659.

6. R. R. Kallman, One-parameter groups of *-automorphisms of $\mathrm{II}_{1}$ von Neumann algebras, Proc. Amer. Math. Soc. 24 (1970), 336-340. MR 40 \#4775.

7. - Unitary groups and automorphisms of operator algebras, Amer. J. Math. 91 (1969), 785-806. MR $40 \# 7825$.

8. D. McDuff, Central sequences and the hyperfinite factor, Proc. London Math. Soc. 21 (1970), 443-461.

9. R. T. Powers, Representations of uniformly hyperfinite algebras and their associated von Neumann rings, Ann. of Math (2) 86 (1967), 138-171. MR 36 \#1989. 
10. L. Pukánszky, Some examples of factors, Publ. Math. Debrecen 4 (1956), 135-156. MR 18, 323.

11. S. Sakai, A symptotically abelian $\mathrm{II}_{1}$ factors, Publ. Res. Inst. Math. Sci. Ser. A4 (1968/69), 299-307. MR $40 \# 1785$.

12. - The theory of $\mathbb{W}^{*}$-algebras, Yale University, New Haven, Conn., 1962.

13. J. T. Schwartz, $W^{*}$-algebras, Gordon and Breach, New York, 1967. MR 38 \#547.

14. J. Tomiyama, On the tensor products of von Neumann algebras, Pacific J. Math. 30 (1969), 263-270. $\quad$ MR $39 \# 7446$.

15. P. Willig, $B(H)$ is very noncommutative, Proc. Amer. Math. Soc. 24 (1970), 204205. MR $40 \# 1789$.

16. - Properties $\Gamma$ and $L$ for type $\mathrm{II}_{1}$ factors, Proc. Amer. Math. Soc. 25 (1970), 836-837. MR 41 \#4266.

17. G. Zeller-Meier, Deux autres facteurs de type $\mathrm{II}_{1}$, Invent. Math. 7 (1969), 235242. MR $40 \# 1788$.

DEPARTMENT OF MATHEMATICS, UNIVERSITY OF PENNSYLVANIA, PHIL ADELPHIA, PENNSYLVANIA 19104 